\title{
The importance of psychiatric disorders in end-stage kidney disease patients
}

\author{
Eun Lee \\ Department of Psychiatry and Institute of Behavioral Medicine, Yonsei University College of Medicine, Seoul, Republic of Korea
}

\section{See Article on Page 231-241}

It is uncertain why psychiatric comorbidities are important for end-stage kidney disease (ESKD) patients. Epidemiological studies have demonstrated that physical health is associated with mental health, and psychiatric problems can adversely affect health by exacerbating the onset, course, complications, and mortality of physical diseases. Research into these relationships has focused on psychiatric conditions that have a relatively high prevalence (such as depression, anxiety, and sleep disturbances) in conjunction with physical diseases that are relevant to public health due to high prevalence or high mortality, such as cardiovascular disease, cancer, organ transplantation, and diabetes [1]. The incidence, severity, and mortality rates of ESKD are also affected by psychiatric problems, such as depression [2]. In this context, studies such as that of Lee et al. [3], who conducted large-scale investigations of the prevalence of psychiatric diseases in ESKD patient populations, are rare.

Studies that have examined the prevalence of comorbidities among patients with both psychiatric problems and physical diseases exhibit wide differences in results due to the varying measurement methods used. Diagnoses of psychiatric disorders are typically concluded by a psychiatrist who determines whether diagnostic criteria have been met through a structured interview [4]. Self-report questionnaires filled out by patients have the potential risk of overestimating the prevalence of psychiatric disorders. For example, a meta-analysis of studies that examined depression among dialysis patients with ESKD as measured through psychiatric interviews reported a prevalence of $23 \%$, whereas the incidence rate of depression as assessed via a questionnaire completed by the patient or the patient's physician was $39 \%$ [5]. Both of these values are much higher than the prevalence in the general population without any physical disease.

Questionnaire-based assessments completed by physicians or patients can evaluate specific symptom domains, such as depression and anxiety, but cannot be considered psychiatric diagnostic tools due to their low specificity. Ideally, the comorbidity rates of psychiatric diseases should be assessed with precise diagnostic interviews conducted by psychiatrists, but few such studies have been published. Thus, well-designed studies that can accurately assess the comorbidity of psychiatric disorders are urgently needed. In a 4-year observational study in which psychiatrists made diagnoses based on criteria after interviewing 508 hemodi-

Received: November 21, 2021; Accepted: November 21, 2021

Correspondence: Eun Lee

Department of Psychiatry, Yonsei University College of Medicine, 50-1 Yonsei-ro, Seodaemun-gu, Seoul 03722, Republic of Korea.

E-mail: leeeun@yuhs.ac

ORCID: https://orcid.org/0000-0002-7462-0144

Copyright (@) 2022 by The Korean Society of Nephrology

(a) This is an Open Access article distributed under the terms of the Creative Commons Attribution Non-Commercial and No Derivatives License (http:// creativecommons.org/licenses/by-nc-nd/4.0/) which permits unrestricted non-commercial use, distribution of the material without any modifications, and reproduction in any medium, provided the original works properly cited. 
alysis patients, the one-year incidence of psychiatric disorders such as dementia, major depression, and delirium was $10.6 \%$ [6]. As it is challenging to diagnose psychiatric diseases, researchers often use questionnaires tailored for several specific psychiatric problems, such as depression and sleep disturbances. Studies that have examined insurance claim codes for psychiatric disorders have similar limitations as those of studies that use questionnaires. Nevertheless, a study by Lee et al. [3] has academic significance in several respects. First, their study was representative of ESKD patients in Korea because the data of more than 100,000 patients were investigated using claims information from a health insurance review and assessment service. Second, although not all psychiatric disorder codes were investigated, a wide range of psychiatric diagnostic codes was examined to study as many comorbidities as possible. Finally, their study was reflective of the psychiatric problems of ESKD patients observed in daily nephrology practice.

Understanding the mechanisms behind the interaction between psychiatric problems and physical diseases is another issue that requires further research. Many retrospective studies have evaluated psychiatric problems in dialysis patients [5]. However, these studies did not identify the direction or mechanisms of interactions and only speculated on the details of possible interactions between psychiatric problems and ESKD. Retrospective studies cannot conclusively determine whether a patient's psychiatric problems affected the onset of ESKD, if their psychiatric problems occurred during the course of ESKD, or whether preexisting psychiatric problems were treated after the onset of ESKD. In addition, the results of a study that examined the progenitor symptoms commonly reported by ESKD patients demonstrated that it can be difficult to identify any such relationships [7]. For example, one study reported that $71 \%$ of ESKD patients experience fatigue, $49 \%$ have anorexia, $47 \%$ complain of pain, $44 \%$ have sleep disturbances, $38 \%$ experience anxiety, and $27 \%$ suffer from depression. Fatigue, loss of appetite, pain, and sleep disturbances also are diagnostic criteria for depression, suggesting that depression might be overestimated in this population.

Studies that have examined the mechanisms by which psychiatric problems affect the course of ESKD are urgently needed. Although most research that has been aimed at determining the mechanisms of psychiatric diseases has occurred over the last 30 years, a number of influences has long been assumed to indirectly affect the course of physical diseases by inducing changes in behavior. For example, depression can indirectly affect the course of ESKD by reducing patient adherence to treatment, dietary management, and exercise regimens, but it also can impact ESKD through direct effects, such as changes in the immune system and alterations in neurotransmitter production. Bautovich et al. [8] proposed a number of reasons for the high prevalence of depression among ESKD patients: 1) lifestyle and socioeconomic factors, such as a sedentary lifestyle or poor diet, which affect both depression and ESKD; 2) inflammatory reactions that impact both diseases or changes that take place in the immune system after the onset of kidney disease; 3 ) depression caused by changes in the immune system due to underlying kidney pathology; 4) worsening of kidney disease due to depression-associated poor adherence to renal disease management regimens; 5) depression caused by fatigue, sleep disturbances, or kidney disease-related pain; and 6) financial difficulties secondary to the costs of kidney disease management and depression caused by loss of job opportunities.

Interactions between depression and physical diseases are believed to be mediated by the immune system, which regulates central nervous system functions via cytokine production and so also affects behavior [9]. Cytokine signals interact with physiological, social, and external environmental conditions through the central nervous system. In addition, cytokines affect the immune system via pathways such as the hypothalamic-pituitary axis or sympathetic nervous system, thereby affecting the course of physical diseases.

It is unknown to what extent comorbid psychiatric problems affect the outcome of ESKD. Few long-term observational studies examining this question have been conducted to date. A 2-year study of 917 incident dialysis patients found strong associations between depressive symptoms and all-cause deaths, cardiovascular disease deaths, and cardiovascular events (adjusted relative hazard: 2.22, 3.27, and 1.68, respectively) [10]. Unfortunately, the results on the effect of psychiatric treatment on ESKD have been unclear. The reality that many physical conditions interfere with active psychiatric treatment and that it remains difficult to follow the course of ESKD over a long period of time has led to skepticism concerning the benefits of active treatment of psychiatric problems in such patients. 
In summary, although ESKD is clinically significant due to its high prevalence and mortality rates, few studies have examined the course of the disease in patients with psychiatric diseases [10]. Additional standardized and systematic studies of a diverse array of psychiatric problems are needed, as are evaluations of the effects of therapeutic interventions on the course of ESKD that examine indicators in the brain, immune system, and kidneys.

\section{Conflicts of interest}

The author has no conflicts of interest to declare.

\section{Funding}

This work was supported by the National Research Foundation of Korea, which is funded by the Ministry of Science, ICT, and Future Planning, Republic of Korea (grant number, 2017R1A2B3008214 to Eun Lee).

\section{ORCID}

Eun Lee, https://orcid.org/0000-0002-7462-0144

\section{References}

1. Sadock BJ, Sadock VA, Ruiz P. Kaplan \& Sadock's synopsis of psychiatry: behavioral sciences/clinical psychiatry. 11th ed.
Philadelphia: Lippincott Williams \& Wilkins; 2015.

2. Kimmel PL, Weihs K, Peterson RA. Survival in hemodialysis patients: the role of depression. J Am Soc Nephrol 1993;4:12-27.

3. Lee MJ, Lee E, Park B, Park I. Mental illness in patients with endstage kidney disease in South Korea: a nationwide cohort study. Kidney Res Clin Pract 2022;41:231-241.

4. First MB. Structured clinical interview for the DSM (SCID). In: Cautin Cautin, Lilienfeld SO, The encyclopedia of clinical psychology. Chichester: Wiley Blackwell; 2015.

5. Palmer S, Vecchio M, Craig JC, et al. Prevalence of depression in chronic kidney disease: systematic review and meta-analysis of observational studies. Kidney Int 2013;84:179-191.

6. Fukunishi I, Kitaoka T, Shirai T, Kino K, Kanematsu E, Sato Y. Psychiatric disorders among patients undergoing hemodialysis therapy. Nephron 2002;91:344-347.

7. Murtagh FE, Addington-Hall J, Higginson IJ. The prevalence of symptoms in end-stage renal disease: a systematic review. $A d v$ Chronic Kidney Dis 2007;14:82-99.

8. Bautovich A, Katz I, Smith M, Loo CK, Harvey SB. Depression and chronic kidney disease: a review for clinicians. Aust N Z J Psychiatry 2014;48:530-541.

9. Irwin MR, Cole SW. Reciprocal regulation of the neural and innate immune systems. Nat Rev Immunol 2011;11:625-632.

10. Boulware LE, Liu Y, Fink NE, et al. Temporal relation among depression symptoms, cardiovascular disease events, and mortality in end-stage renal disease: contribution of reverse causality. Clin J Am Soc Nephrol 2006;1:496-504. 